\title{
Late Worsening of COVID-19 Pneumonia: Successful Treatment with Ruxolitinib and Steroid
}

\author{
Mauro Betelli, Fabio De Stefano, Alberto Tedeschi \\ Medicina Generale, Bolognini Hospital, ASST Bergamo Est, Seriate, Italy
}

Doi: 10.12890/2020_001938 - European Journal of Case Reports in Internal Medicine - ๑ EFIM 2020

Received: 07/08/2020

Accepted: $11 / 09 / 2020$

Published: $14 / 10 / 2020$

How to cite this article: Betelli M, De Stefano F, Tedeschi A. Late worsening of COVID-10 pneumonia: successful treatment with ruxolitinib and steroid. EJCRIM 2020;7: doi:10.12890/2020_001938.

Conflicts of Interests: The Authors declare that there are no competing interests.

This article is licensed under a Commons Attribution Non-Commercial 4.0 License

\section{ABSTRACT}

We describe the case of a patient hospitalized for the second time in a month due to delayed worsening of lung lesions in COVID-19 infection without bacterial superinfection. He was treated with hydroxychloroquine, IV dexamethasone and ruxolitinib with rapid improvement of respiratory failure; 1 month after the second discharge, maintaining low-dose oral prednisone, lung consolidations were significantly reduced on control CT.

\section{LEARNING POINTS}

- Modulation of immune over-response in late phases of COVID-19 can influence global outcome.

- Ruxolitinib and IV steroids can reverse the inflammatory process and lung lesions.

\section{KEYWORDS}

COVID-19, ruxolitinib, Janus kinase inhibitors

\section{INTRODUCTION}

The clinical picture of coronavirus disease 2019 (COVID-19) caused by severe acute respiratory syndrome coronavirus 2 (SARS-CoV-2) is variable, depending on cumulative viral exposure and both the innate and adaptive immune response ${ }^{[1]}$. Late phases of COVID-19 pneumonia can be characterized by a hyper-inflammation with a cytokine storm leading to alveolar damage ${ }^{[2]}$. Treatments are therefore aimed at reducing inflammation and include steroids, cytokine antagonists- such as the anti-interleukin-6 receptor antibody tocilizumab and the interleukin-1 receptor antagonist anakinra - and Janus kinase (JAK) inhibitors ${ }^{[3]}$. Ruxolitinib (Jakavi ${ }^{\circledR}$ ) is a potent inhibitor of JAK1 and 2, which is currently approved for the treatment of myeloproliferative neoplasms such as primary myelofibrosis and polycythaemia vera, but it is also effective in disorders where cytokines play a pivotal role, such as steroid-refractory graft-versus-host disease and haemophagocytic lymphohistiocytosis. The efficacy of ruxolitinib relies on a powerful anti-inflammatory action by inhibition of the JAK/ STAT (signal transducer and activator of transcription) pathway activated by cytokines. In view of its anti-inflammatory activity, the compassionate use of ruxolitinib in the treatment of COVID-19 was approved by the Italian Medicines Agency (AIFA) on April 2, 2020. We hypothesized a synergistic anti-inflammatory effect of systemic steroids and JAK inhibition, and we decided to use a combined treatment with dexamethasone and ruxolitinib in our patient with delayed lung damage presumably due to the release of proinflammatory cytokines and growth factors induced by infection with SARS-CoV-2. 
CASE DESCRIPTION

A 67-year-old man was admitted to a Bergamo district hospital because of interstitial pneumonia during the COVID-19 outbreak in March 2020.

The patient had a history of hypertension; medications included amlodipine and doxazosin. There were no known allergies.

At admittance, the nasopharyngeal swab was positive for SARS-CoV-2 and chest x-ray showed diffuse areas of emphysema and pulmonary infiltrates consistent with a COVID-19 diagnosis. The patient was treated with hydroxychloroquine, azithromycin and ceftriaxone; lowflow nasal cannula oxygen was administered with no need for ventilatory support. Atrial fibrillation with a normal heart rate was also found during routine electrocardiography; anticoagulation therapy with enoxaparin and low-dose bisoprolol were started. After 28 days of hospitalization, the patient was discharged; he complained of a mild dyspnoea on exertion, 2 nasopharyngeal swabs were negative and lung lesions were improved with initial basal fibrosis on a computed tomography (CT) scan. Enoxaparin was substituted with apixaban.

One week after discharge from the hospital, the patient attended the emergency department of our hospital complaining of dyspnoea and fever. Blood pressure was 120/80 mmHg, heart rate 103 beats per minute, respiratory rate 28 breaths per minute, temperature $37.7^{\circ} \mathrm{C}$ and oxygen saturation was $96 \%$ while he was receiving supplemental oxygenation through a Venturi mask at a rate of 15 litres per minute. On examination, he appeared in mild respiratory distress and breath sounds were decreased throughout all fields with rales at the bases; heart rhythm was irregular, there were no signs of congestive heart failure. A new chest CT scan showed ground-glass opacities and segmental consolidations with more extensive lung involvement and mild right pleural effusion; on electrocardiography, atrial fibrillation with a high ventricular rate was present; the nasopharyngeal swab was negative; blood and urine cultures were negative; Legionella and pneumococcal urinary antigens were negative. Blood tests revealed inflammation: haemoglobin was $11.5 \mathrm{~g} / \mathrm{dl}$, the white cell count $8,010 / \mu \mathrm{l}$

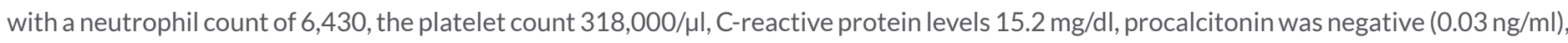
interleukin- 6 was $57 \mathrm{pg} / \mathrm{ml}$ (normal value $<6.0 \mathrm{pg} / \mathrm{ml}$ ) and lactate levels were $2.56 \mathrm{mg} / \mathrm{dl}$; creatinine levels $1.6 \mathrm{mg} / \mathrm{dl}$, hepatic transaminases were within the normal range. Blood gas analysis in ambient air showed hypocapnic respiratory failure: $\mathrm{pH}^{7.45}, \mathrm{pCO}_{2} 29$ mmHg, $\mathrm{pO}_{2} 69$ $\mathrm{mmHg}, \mathrm{HCO}_{3} 22 \mathrm{mmol} / \mathrm{l}, \mathrm{O}_{2}$ saturation $94 \%$.

The patient was hospitalized and evaluated by anaesthesiologists: invasive ventilation was excluded due to the high risk of emphysematous bullae perforation and self-prone positioning was suggested. Empirical treatment with intravenous piperacillin/tazobactam $4.5 \mathrm{~g}$ tid and oral azithromycin $500 \mathrm{mg}$ qd was started.Suspecting a delayed immune over-response ${ }^{[1]}$, anti-inflammatory/antiviral treatment was added with hydroxychloroquine $200 \mathrm{mg}$ qd (with a first day load of $400 \mathrm{mg}$ bid), IV dexamethasone (20 mg qd for 5 days, then $10 \mathrm{mg}$ qd for 5 days, then oral prednisone $25 \mathrm{mg}$ qd) and the JAK inhibitor ruxolitinib $5 \mathrm{mg}$ bid for 14 days; apixaban was substituted with enoxaparin. An echocardiogram was obtained with evidence of preserved systolic function. After 2 weeks, lung consolidations were no longer present on chest radiography, acute phase reactants were normalized and oxygen supplementation was suspended; 23 days after admittance, the patient was discharged with tapering steroid therapy up to a maintenance dose of $5 \mathrm{mg}$ qd of oral prednisone.

One month after the second discharge, the patient was asymptomatic and underwent a third chest CT scan that showed diffuse reduction of parenchymal consolidations with persistence of ground-glass opacities and interlobular septal thickening (see Fig. 1); ongoing therapy was confirmed until the next outpatient follow-up in 3 months.
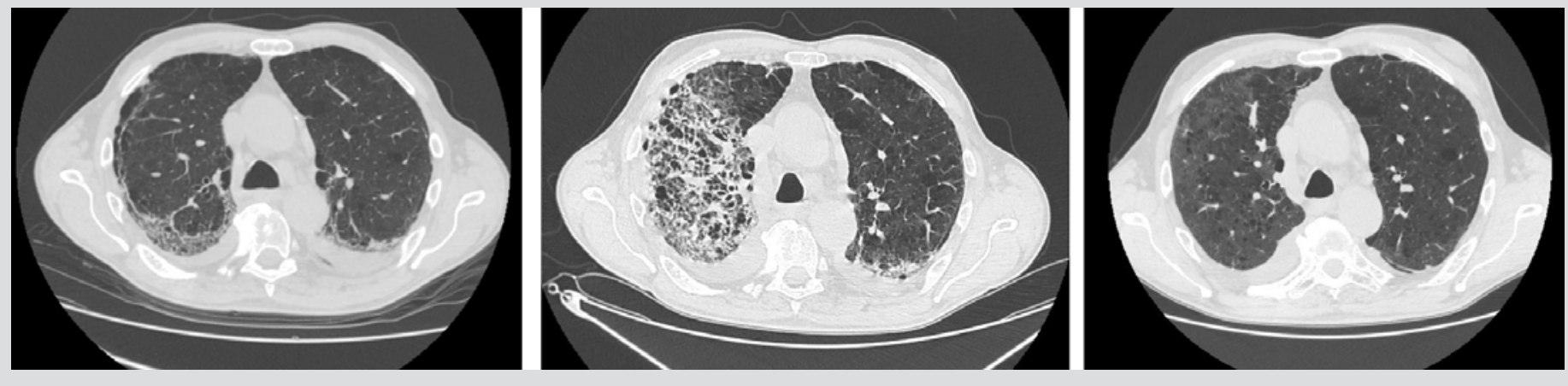

Figure 1. Chest CT showing COVID-19 pneumonia during the initial and second hospitalizations, and at follow-up. Left: Chest CT at day 26 from symptoms onset (first hospitalization). Center: Chest CT at day 25 (second hospitalization). Right: Chest CT at day 78 (outpatient control) 
DISCUSSION

In the case described here, lung injury occurred approximately 35 days after the onset of COVID-19 symptoms, in the absence of active viral replication and bacterial superinfection. It is conceivable that respiratory failure was due to the strong immune/inflammatory response elicited by the virus in the second phase of the disease, as evidenced by the prominent role played by the interleukin- $6 \mathrm{JAK}$ inhibitor, acting to restrain the signalling of this cytokine, which seems to be effective in inflammatory process reversal ${ }^{[4,5]}$, especially in synergy with highdose IV steroids. This case report supports the possible beneficial effects of combined treatment with systemic steroids and ruxolitinib on late lung damage induced by SARS-CoV-2 infection. This report has some limitations due to its anecdotal nature, the lack of a proper control and the contemporaneous use of 2 immunomodulating and anti-inflammatory drugs. However, we believe that it would be worthwhile to evaluate the combined effect of steroids and JAK inhibitors in a controlled clinical trial.

\section{REFERENCES}

1. Matricardi PM, Dal Negro RW, Nisini R. The first, holistic immunological model of COVID-19: implications for prevention, diagnosis, and public health measures. Pediatr Allergy Immunol 2020; 31(5): 454-470.

2. Siddiqi HK, Mehra MR. COVID-19 illness in native and immunosuppressed states: a clinical-therapeutic staging proposal. J Heart Lung Transplant 2020;39(5):405-407.

3. Portsmore S, Tran Nguyen TN, Beacham E, Neelakantan P. Combined IL-6 and JAK-STAT inhibition therapy in COVID-19 related sHLH, potential game changer. Br J Haematol 2020;190(4):525-528

4. Capochiani E, Frediani B, lervasi G, Paolicchi A, Sani S, Roncucci P, et al. Ruxolitinib rapidly reduces acute respiratory distress syndrome in COVID-19 disease. Analysis of data collection from RESPIRE protocol. Front Med (Lausanne) 2020;7:466.

5. La Rosée F, Bremer HC, Gehrke I, Kehr A, Hochhaus A, Birndt S, et al. The Janus kinase 1/2 inhibitor ruxolitinib in COVID-19 with severe systemic hyperinflammation. Leukemia 2020;34(7):1805-1815. 